\title{
Socioeconomic impacts of COVID-19 in low-income countries
}

\author{
Anna Josephson ${ }^{1}$, Talip Kilic $\circledast^{2}$ and Jeffrey D. Michler ${ }^{1}{ }^{1 凶}$
}

The emergence of SARS-CoV-2 and attempts to limit its spread have resulted in a contraction of the global economy. Here we document the socioeconomic impacts of the pandemic among households, adults and children in low-income countries. To do so, we rely on longitudinal household survey data from Ethiopia, Malawi, Nigeria and Uganda, originating from pre-COVID-19 face-to-face household surveys plus phone surveys implemented during the pandemic. We estimate that 256 million individuals $-77 \%$ of the population-live in households that have lost income during the pandemic. Attempts to cope with this loss are exacerbated by food insecurity and an inability to access medicine and staple foods. Finally, we find that studentteacher contact has dropped from a pre-COVID-19 rate of $96 \%$ to just $17 \%$ among households with school-aged children. These findings can inform decisions by governments and international organizations on measures to mitigate the effects of the COVID-19 pandemic.

S ARS-CoV-2 is a major global threat to human health, with 102 million cases and 2.2 million deaths worldwide ${ }^{1}$ as of 2 February 2021. Governments have sought to limit the spread of the virus and mitigate the negative health outcomes of the disease through various policy measures that have limited travel, imposed quarantines and lockdowns, and closed businesses and schools ${ }^{2,3}$. In addition to the economic burden of COVID-19-related morbidity and mortality, there have been profound economic impacts, with the global economy projected to shrink ${ }^{4}$ by $8 \%$. To date, the greatest health and economic burden has been borne by the Americas and Europe ${ }^{1}$. However, recent evidence indicates that low-income countries, with their limited health system capacities, are likely to suffer infection and mortality rates similar to or greater than those currently suffered by high-income countries ${ }^{5}$. While research on the health impacts of COVID-19 in low-income countries is rapidly emerging ${ }^{6}$, there is limited evidence on the socioeconomic impacts of the pandemic ${ }^{7}$. The evidence that exists relies primarily on pre-COVID-19 macroeconomic data and simulation models to forecast potential future scenarios on the basis of assumptions about the disease spread $^{8}$. By contrast, we rely on direct measurements of socioeconomic indicators to document the implications of the pandemic for households, adults and children living in low-income countries, as well as the actions that households are taking to mitigate these impacts.

An acute challenge emerging from the global pandemic is how individuals and communities are to strike the balance between the health benefits and the economic costs of managing the spread of the virus ${ }^{2,3}$. Even in high-income countries, which tend to be data rich in terms of health and economic information, striking this balance frequently proves politically difficult. By contrast, low-income countries, which tend to be resource-constrained, are data poor in terms of reliable and timely information on the spread of SARS-CoV-2 and on the health and economic impacts of anti-contagion policies. Our objective is to directly measure, at the household, adult and child levels, the socioeconomic impacts of the pandemic and the policies implemented to slow the spread of the virus. While we make no claims of causal identification arising from our statistical analysis, most of the outcomes we examine arise from survey questions that ask directly about how the pandemic and the accompanying restrictions have affected the respondent and her or his household. Our goal is to learn from how individuals cope with the socioeconomic effects of the virus. This can inform decisions by governments and international aid organizations regarding how best to mitigate the persisting effects of the COVID-19 pandemic.

\section{Results}

Our findings are based on longitudinal data from high-frequency phone surveys conducted in Ethiopia, Malawi, Nigeria and Uganda with support from the World Bank. Starting in May 2020, and continuing every month thereafter, the phone surveys aim to interview a nationally representative sample of households. They follow up households that had been previously been interviewed face-to-face, prior to the COVID-19 pandemic. Both pre-COVID-19 face-to-face surveys and post-outbreak phone surveys have been supported under the World Bank Living Standards Measurement Study (LSMS)-Integrated Surveys on Agriculture (ISA) initiative.

The pre-COVID-19 LSMS-ISA-supported surveys that serve as sampling frames for the phone surveys were designed to be representative at the national, regional and urban-and-rural levels. These surveys include the Ethiopia Socio-economic Survey 2018-2019, Malawi Integrated Household Panel Survey (IHPS) 2019, Nigeria General Household Survey (GHS) Panel 2018/19, and Uganda National Panel Survey 2019/20. In Ethiopia, Malawi and Uganda, the phone survey attempted to call all LSMS-ISA households for whom at least one phone number was available either for a household member or for a reference individual. In Nigeria, a national subsample was drawn from the set of LSMS-ISA households with phone numbers. The anonymized survey data and documentation are accessible through the World Bank Microdata Library ${ }^{9-12}$ and are comparable across countries, based on the template questionnaires and the phone survey sampling guidelines (see Methods and Supplementary Information for more information on the sampling frame).

We directly measure the effects of the pandemic on 10,855 households across the four countries, as well as how these households

'Department of Agricultural Economics, University of Arizona, Tucson AZ, USA. 2Development Data Group, World Bank, Washington, DC, USA.

凶e-mail: jdmichler@arizona.edu 
attempt to cope with these experiences. We use well-established methods $s^{13-15}$ to recalibrate pre-COVID-19 sampling weights and correct them for potential selection bias associated with not interviewing households that do not own mobile phones or that cannot be reached despite repeated call attempts. In turn, we are able to provide estimates of the total number of households and individuals, including adults and children, associated with any of the reported outcomes, thanks to the selection-bias corrections and the post-stratification adjustments that ensure that the population estimates implied by the sampling weights in each country match with the official population projections for that country at the time of the phone surveys (see Methods for details on the estimation of phone survey sampling weights). We then use econometric methods common to the field ${ }^{16,17}$ to estimate heterogeneity in effects across (1) countries, (2) rural and urban sectors, (3) pre-COVID-19 wealth, and (4) time (see Methods). In the main body of the paper we present visualizations of the estimated magnitudes of the effects, placing all statistical tests, confidence intervals and supporting results in the Supplementary Material.

Tracking how people differentially experience the COVID-19 pandemic can enable governments and policymakers to better understand the circumstances faced by their citizenry and to make data-driven, informed policy decisions. The need to understand the contemporaneous impacts and coping strategies of households is and will continue to be important for low-income countries, as they are likely to lack access to a vaccine for longer than high-income countries ${ }^{18,19}$. The longitudinal data collected through the high-frequency phone surveys cultivate this understanding by documenting trends within a month or two of their occurrence.

\section{Awareness and knowledge of COVID-19}

With the global spread of the SARS-CoV-2 virus, numerous low-income countries followed worldwide trends by declaring states of emergency, issuing stay-at-home orders, closing schools and imposing curfews ${ }^{2,20}$. Considering the countries in our dataset, Ethiopia closed schools and suspended public gatherings on $16 \mathrm{March}^{21}$ and declared a five-month state of emergency on 8 April, which included closing non-essential business, suspending flights, closing land borders and limiting cross-country and inter-city public transport ${ }^{22}$. The Nigeria response occurred primarily at the state level, with most states closing schools and suspending large gatherings such as religious services, between 18 and $24 \mathrm{March}^{23}$. Though no state of emergency or lockdown was declared at the federal level, by early April most states had closed all non-essential businesses, suspended public transportation and closed state borders ${ }^{24}$. The Government of Uganda announced that it was closing schools, churches, limiting large gatherings and closing the international border on $18 \mathrm{March}^{25}$. By $30 \mathrm{March}$, Uganda had instituted a country-wide shutdown, closing all non-essential businesses and suspending public and private transport ${ }^{26}$. In Malawi, the President declared a state of disaster on 20 March, closing schools and limiting the size of public gatherings ${ }^{27}$. Malawi attempted to issue stay-at-home orders on $14 \mathrm{April}^{28}$, but the country's High Court barred the government from implementing and enforcing the lockdown ${ }^{29}$. As a result, Malawi-unlike the other countries in our study-instituted no stay-at-home order or lockdown, although the government did encourage citizens to stay at home. This heterogeneity in government responses is reflected in the data (Fig. 1a). The legal wrangling in Malawi appears to have contributed to individuals in Malawi being significantly less aware of government and local authority actions to curb the spread of the virus (see Supplementary Table 1).

This divergent path observed in Malawi is also related to individual knowledge of COVID-19 and the adoption of behaviours to limit the spread of SARS-CoV-2. In Ethiopia, Nigeria and Uganda, knowledge about measures that can be adopted to reduce the risk of contracting the virus is extremely high (Fig. 1b). In Uganda, we estimate that more than $90 \%$ of individuals are familiar with all six mitigation measures asked about in the survey, while in Ethiopia and Nigeria at least $80 \%$ of individuals are familiar with five of the six measures. By comparison, in Malawi, handwashing with soap is the only mitigation strategy with which at least $80 \%$ of individuals are familiar. Malawians are significantly less knowledgeable about the other five mitigation strategies than respondents in Ethiopia, Nigeria or Uganda (Supplementary Table 2).

The heterogeneity in knowledge between Malawi and the other three countries is reflected in changing behaviour related to the virus (Fig. 1c). In Ethiopia, Nigeria and Uganda, we estimate that adoption of handwashing is nearly universal, as is avoidance of physical contact in the week before the interview. Even in Malawi, we estimate that around $90 \%$ of individuals wash their hands more frequently than before and avoid physical contact. Yet, significantly fewer individuals in Malawi report adapting their behaviour to help reduce the spread of the virus relative to respondents in Ethiopia, Nigeria and Uganda (Supplementary Table 3). In Uganda and Malawi, where we have behavioural data over time, we show that the number of people continuing to wash hands and avoid crowds decreased significantly over the period from June to August 2020 (Supplementary Table 4).

The lower levels of knowledge and behavioural change in Malawi are further manifested in a higher prevalence of beliefs in unproven, disproven or false claims about the virus and the disease (Fig. 1d). These data exist only for Malawi and Uganda. Malawians are significantly more likely than Ugandans to believe that Africans are immune to the virus, that children are not affected by the virus, and that the disease is no different than the common flu. While Malawians are significantly more likely to hold several of these false beliefs (Supplementary Table 5), the number of people in Uganda lacking accurate information about SARS-CoV-2 is higher than the number in Malawi, due to Uganda's larger population. We estimate that 3.1 million individuals in Malawi believe that the virus is just the common flu, the most common false belief. In Uganda, the most common false belief is that the virus cannot survive warm weather, with an estimated 6.1 million individuals subscribing to this disproven claim (Supplementary Table 6). An estimated 1.3 million to 3.3 million adults in each country believe the other false claims. These represent substantial shares of the population in each country and reveal the continued need for clear and accurate messaging about COVID-19 to avoid the rapid and far-reaching spread of information of questionable quality ${ }^{30}$.

\section{Economic impacts and food security}

As countries declared states of emergency, issued stay-at-home orders and advised avoidance of social gatherings, hundreds of millions of individuals in low-income countries found themselves out of work, in both the formal and informal labour markets ${ }^{31}$. Correspondingly, households have lost income across a variety of sources (Fig. 2a). The surveys asked households whether their income had decreased since the outbreak in mid-March. To have lost income from a particular source, the household must have previously received income from that source; that is, our estimates of losses are conditional on having received income from a given source in the previous 12 months (Supplementary Table 7). We estimate that 256 million individuals, $77 \%$ of the population across the four countries, live in households that have lost income since the onset of the pandemic (Supplementary Table 8). It should be noted that not all lost income may be directly attributable to the pandemic, and so this number should be considered an upper bound. Considering country-level heterogeneity in income loss, Ethiopian households are significantly less likely to have lost income compared with those from the other three countries. There is no evidence that the share of households that have lost income are different across 
a

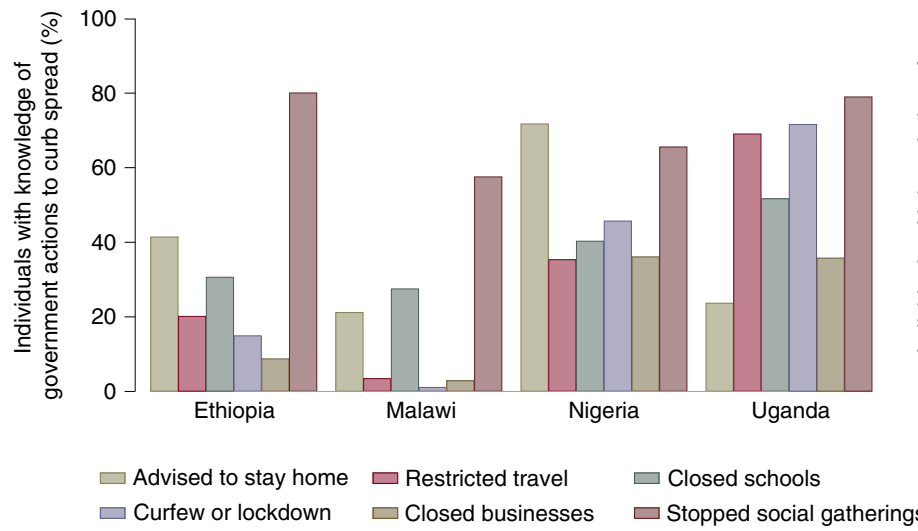

C

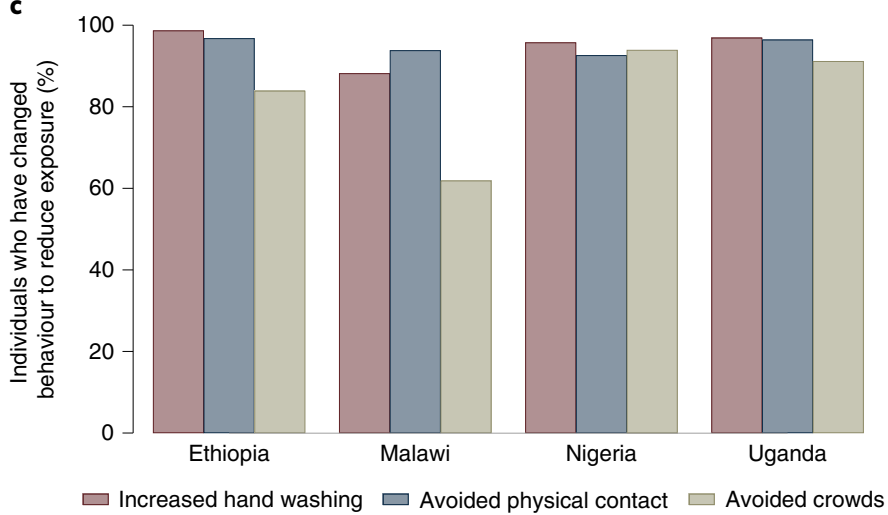

b

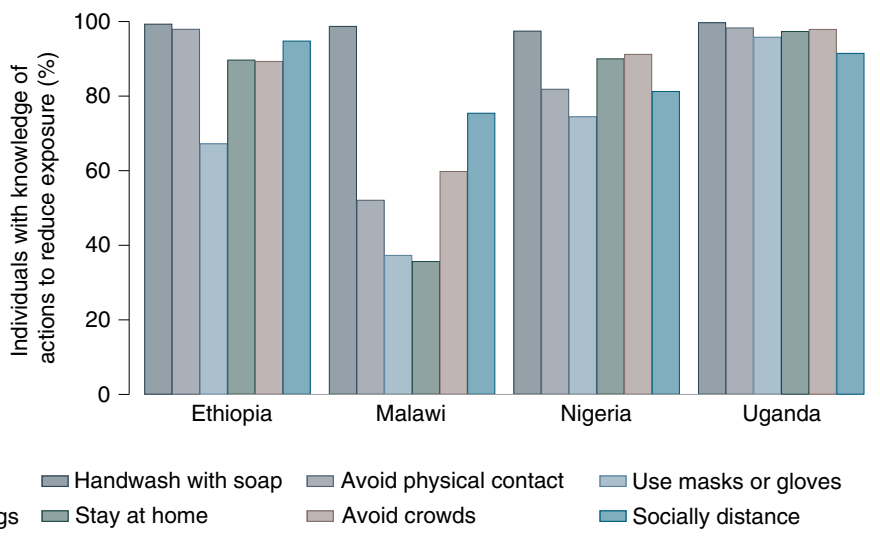

d

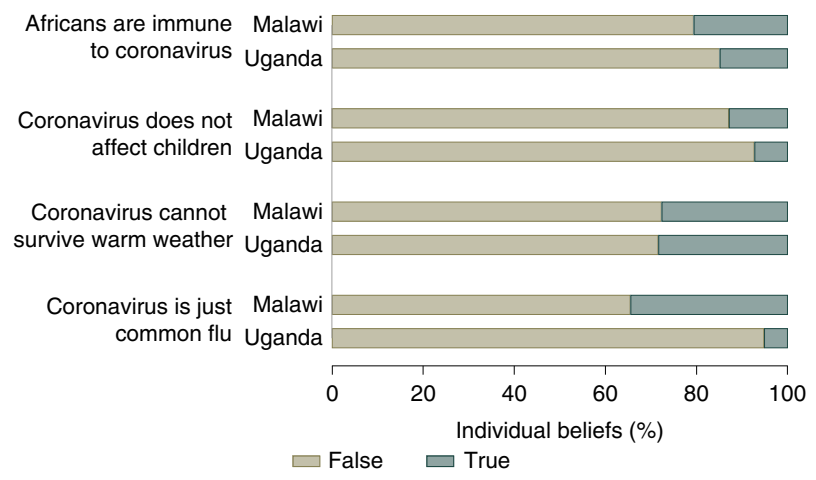

Fig. 1 | Knowledge of COVID-19 restrictions, behaviours and false beliefs. a, Percentage of individuals with knowledge regarding government actions undertaken to curb the spread of COVID-19 $(n=9,158)$. b. Percentage of individuals with knowledge of actions that an individual can take to reduce exposure to COVID-19 ( $n=9,178)$. c, Percentage of individuals reporting a change in behaviour in the previous $7 \mathrm{~d}(n=9,186)$. d, Percentage of individuals in Malawi and Uganda with beliefs about common misconceptions regarding coronavirus $(n=3,867)$.

Malawi, Nigeria and Uganda (Supplementary Table 9). In the majority of cases, we find no credible evidence of heterogeneity in income loss between rural and urban households (Supplementary Table 10). This suggests that income losses have been borne similarly across the rural and urban populations, both within and across countries.

While households have lost income from a variety of sources, we focus on losses to non-farm enterprises (NFEs), as they are the income sources likely to be most affected by shutdown and lockdown orders. An estimated 35\% of households across all four countries operated an NFE before the pandemic. Of these, a majority report that revenue is down, compared with pre-COVID-19 levels in February (Fig. 2b). As with income losses, not all lost revenue from NFEs can be directly attributed to the pandemic. Revenue loss varies by country, and we estimate that Ugandans with NFEs are significantly less likely to experience revenue losses than their Ethiopian, Malawian and Nigerian counterparts. Tracking these dynamics over time shows that some NFEs are recovering revenue relative to previous rounds of the survey. Across all countries, significantly higher numbers of individuals report making the same or higher revenue than in the first round of interviews. However, in Ethiopia and Nigeria, the estimated differences between the second and third rounds are not significant (Supplementary Table 11). This suggests the lack of a clear trajectory in recovery of household businesses over the coming months.

Estimating these income and revenue losses is important as they may have reverberating influence on other elements of the household economy, in particular food security. We estimate the prevalence of food insecurity among the adult population, as measured by the Food Insecurity Experience Scale (FIES) (Methods). On the basis of our estimates, $60 \%$ of the adult population, representing more than 98 million adults across all four countries, experience moderate or severe food insecurity. Severe food insecurity alone affects an estimated 36 million adults, or $22 \%$ of the adult population (Supplementary Table 12). It should be noted that these are estimates, and are not themselves indicative of a change in food insecurity since the beginning of the global pandemic. To examine how food insecurity may vary across the wealth spectrum, we used the pre-COVID-19 LSMS-ISA survey data to calculate household annual per capita income and to generate consumption quintiles (Methods). Examining heterogeneity across consumption quintiles, poorer households (in lower quintiles) suffer significantly higher prevalence of food insecurity (Fig. 2c). Further, there is significant heterogeneity in the prevalence of food insecurity across countries (Supplementary Table 13). Nigeria suffers the highest prevalence of food insecurity - an estimated $76 \%$ of adults (63 million) are moderately or severely food insecure. This is followed by Malawi with $68 \%$ of adults (6.2 million), Ethiopia with $44 \%$ of adults (22 million) and Uganda with $33 \%$ of adults (6.9 million) with moderate or severe food insecurity. While these estimates do not reflect changes in food insecurity since the pandemic, the current status of food insecurity in these countries is of interest to governments, policymakers and other researchers. The one country from which we have data that enable us to compare a household's current level of food insecurity to its pre-COVID-19 level is Nigeria (Extended Data Fig. 1); these data show a statistically significant 

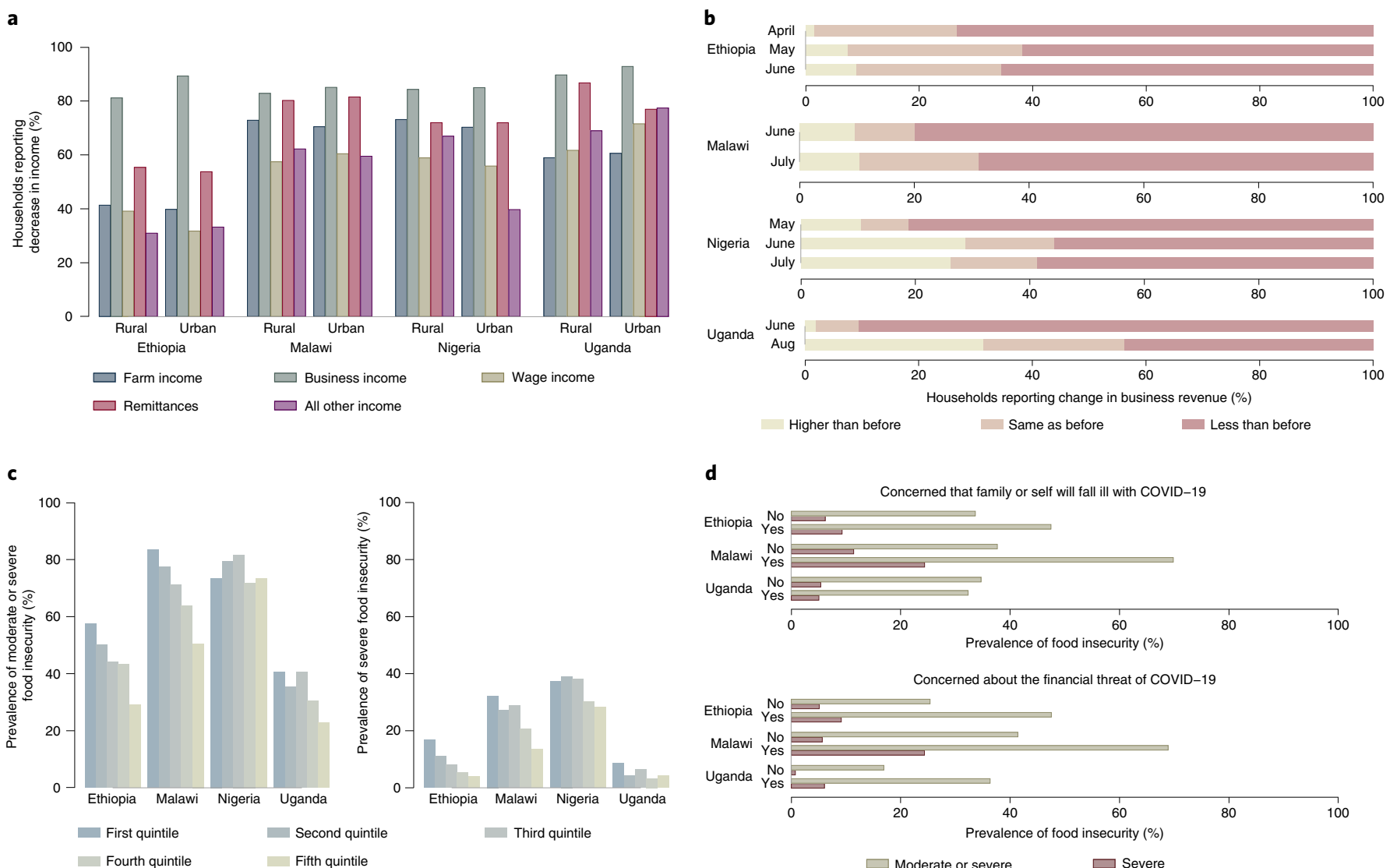

Fig. 2 | Household income, food insecurity and concerns about COVID-19. a, Percentage of households reporting loss of income sources, by country and rural or urban residence $(n=8,832)$. b. Percentage of households reporting change in business revenue, by country and round of survey $(n=4,357)$. c, Prevalence of moderate and/or severe food insecurity among adult individuals, by country and pre-COVID-19 household annual per capita consumption quintile $(n=8,711)$. d, Prevalence of food insecurity among adult individuals, by country and COVID-19-related concerns linked to health and finance $(n=8,816)$.

increase in food insecurity among households since the onset of the pandemic (Supplementary Table 14). While we lack direct evidence for Ethiopia, Malawi and Uganda, the significant increase in food insecurity in Nigeria combined with evidence from the European Union and the United States ${ }^{32-34}$ suggests that households have been experiencing a high prevalence of both moderate and severe food insecurity since the pandemic began.

The spread of COVID-19, combined with the resulting loss of income and revenue from NFEs, has led to high levels of concern among households regarding their health and financial wellbeing. An estimated 257 million individuals (78\%) are concerned that someone will fall ill with COVID-19 and 292 million individuals (88\%) are concerned about financial threats related to COVID-19 (Supplementary Table 15). We divide households on the basis of their response regarding being concerned about illness or finances and chart the prevalence of food insecurity within each group. Higher prevalence of food insecurity is correlated with concerns about household health and financial status, especially in Malawi (Fig. 2d). There is substantial heterogeneity in the prevalence of food insecurity on the basis of reported concern over health and finances. Those concerned about the financial threat of COVID-19 have a significantly higher prevalence of food insecurity than those unconcerned about financial threats (Supplementary Table 16). At the same time, we find no credible difference in the prevalence of food insecurity on the basis of whether or not a household is concerned about health-related impacts. Such variation does not suggest any clear pattern among those who are concerned about finances versus health and why, although COVID-19-related concerns weigh heavily on individuals in many contexts.

\section{Access to basic necessities and education}

Since the closure of schools and the issuance of emergency stay-at-home orders, households have suffered a variety of economic shocks, some attributable to these closures and others simply part of the inherently risky lives of many households in low-income countries. These shocks include job loss, business closure, disruption of farming activity, rising input prices, falling output prices, increasing food prices or illness or death of an income earner. In total, an estimated 25 million households (around 42\%) have suffered one or more of these shocks since mid-March. This ranges from $26 \%$ of households ( 5 million) in Ethiopia to $56 \%$ of households (15 million) in Nigeria (Supplementary Table 17). Households have adopted a number of strategies to try to cope with these shocks (Fig. 3a). To adopt a coping strategy, a household must have experienced a shock since mid-March; that is, our estimates for the adoption of each coping strategy are conditional on exposure to a shock. The specific coping strategy that households adopt is context-dependent, but the estimated number of households adopting some strategy is indicative of the scale of economic loss (Supplementary Table 18). These strategies include living off savings (12 million households), selling assets (3.5 million), reducing food or non-food consumption (21 million and 6.5 million, respectively), receiving help from family (8.1 million), and receiving government assistance (4.3 million), although there is significant heterogeneity 

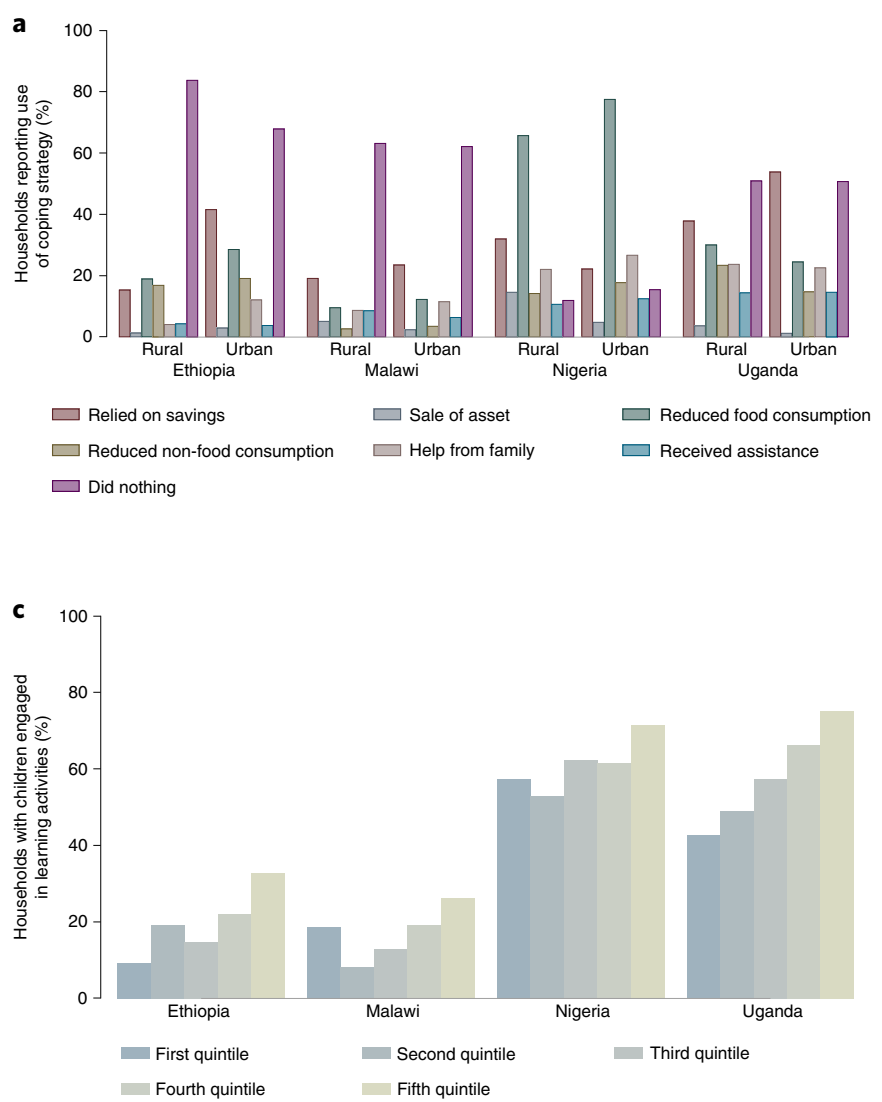
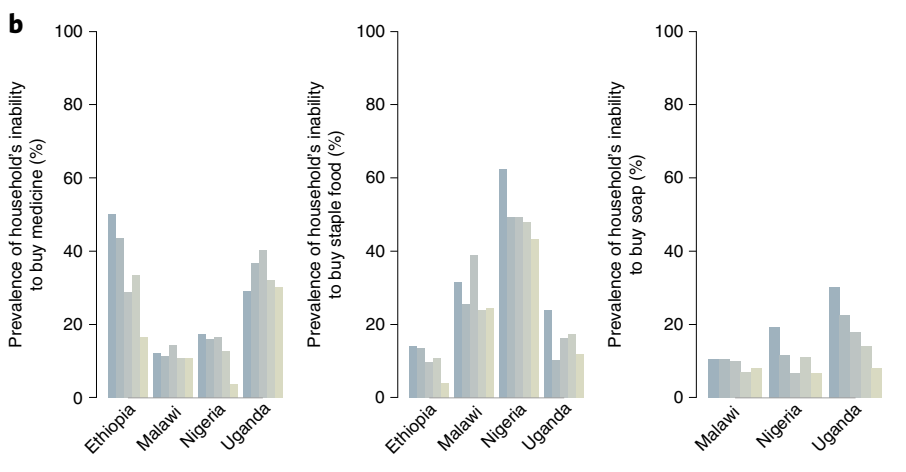

$$
\text { First quintile }
$$
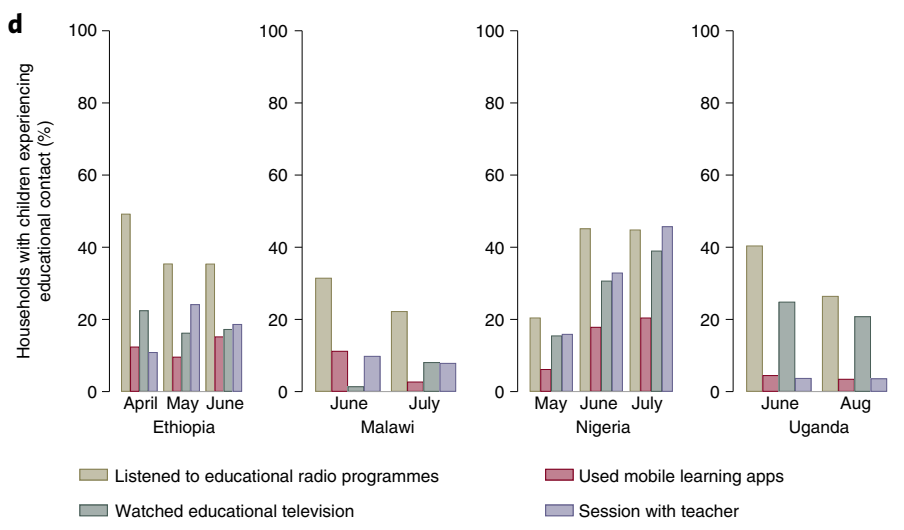

Fig. 3 | Household coping strategies and access to basics necessities. a, Percentage of households reporting use of coping strategy, by country and rural or urban residence $(n=9,614)$. b. Share of households reporting a lack of access to medicine, staple food and soap, by country and pre-COVID-19 household annual per capita consumption quintile $(n=9,120)$. c, Percentage of households with school-aged children experiencing educational contact, by country and survey round $(n=6,605)$. d, Percentage of households with children engaged in learning activities, by country and pre-COVID-19 household annual per capita consumption quintile $(n=4,109)$.

across countries (Supplementary Table 19). There is also heterogeneity between rural and urban households: we estimate that rural households are significantly more likely to rely on the sale of assets, whereas urban households are significantly more likely to reduce food consumption or rely on friends and family to cope with shocks experienced since the outbreak of the pandemic (Supplementary Table 20).

The challenges of coping with lost income are exacerbated by the inability to purchase basic necessities (Fig. 3b). Conditional on the households having sought to purchase medicine, an estimated $29 \%$ are unable to buy what they need. Access to staple foods is similarly limited, with an estimated $30 \%$ of households unable to purchase the staple. Soap is relatively more available, with only an estimated $12 \%$ of households being unable to purchase soap when they needed it (Supplementary Table 21). Again, it should be noted that these estimates represent an upper bound as, even in the absence of the pandemic, there would be a non-zero number of households that could not access medicine, staple foods or soap. Households report a number of different reasons for this lack of access. For soap and medicine, the vast majority $(80 \%)$ report that their lack of access is due to a decrease in income since mid-March. For staple foods, about $50 \%$ report an increase in prices, while about $40 \%$ report a decrease in income as the reason for their lack of access. This lack of access to basic necessities varies by consumption quintile. Households that were in the lowest consumption quintile before the pandemic are significantly less likely to be able to access medicine than households that were in the highest consumption quintile
(Supplementary Table 22). Similarly, households in the lowest consumption quintile are less likely to have access to staple foods and soap than households in the other quintiles. The disproportionate burden of the disease on the poorest has been noted previously ${ }^{35}$, and the distribution of the economic burden has been speculated on $^{36}$ - here we provide direct evidence of this playing out in the past few months among the surveyed households.

Before the spread of SARS-CoV-2, the global community had made substantial progress towards completing Millennium Development Goal 2: universal primary education. As of 2015, primary school enrolment rates in low- and middle-income countries $^{37}$ had reached $91 \%$. On the basis of our data, children from an estimated $96 \%$ of households with school-aged children attended school before the outbreak. Following the outbreak and school closures, the incidence of school-aged children who were previously attending school engaging in any learning activity fell to an estimated $46 \%$. We estimate the incidence of student-teacher contact in the week before the surveys at just $17 \%$. Student-teacher contact encompasses communication through a variety of media, including SMS, online video communication tools, email, mail, telephone and/or WhatsApp. These estimates amount to 68 million school-aged children across the four countries not being engaged in education during this period (Supplementary Table 23). As with access to basic necessities, there is a disproportionate lack of access to education among poorer households (Fig. 3c). Children from households in the top $40 \%$ of the pre-COVID-19 consumption distribution were significantly more likely to be engaged in a learning 
activity since the outbreak than children from households in the lower quintiles (Supplementary Table 24).

The loss of educational contact is widespread and likely to have long-term consequences, although we find evidence that households are using technologies such as radio, television and mobile learning apps to mitigate educational losses (Fig. 3d). While millions of children in these countries are participating in various types of educational contact, millions more are unable to do so. We estimate the share of households with school-aged children using these technologies to be below 50\%. This may have short-term consequences for adults trying to return to work, as well as long-term consequences for children's educational attainment. There is good news, however, in that our estimates show engagement with several of these technologies to be increasing over time. In Ethiopia, Malawi and Nigeria, there have even been significant increases in student contact with teachers in recent months (Supplementary Table 25).

\section{Discussion}

In order to formulate policies and target resources at mitigating the adverse health and economic impacts of the COVID-19 pandemic, governments, international organizations, non-governmental organizations and other stakeholders need reliable and timely data and estimates of the circumstances faced by individuals and households.

The results presented here provide early documentation of the socioeconomic impacts of the COVID-19 pandemic in low-income countries. Governments in these countries were already facing complex and mutually reinforcing development challenges before the pandemic, and the widespread availability of a COVID-19 vaccine in the region is not expected until $2022^{18,19}$. Our analysis is focused on Ethiopia, Malawi, Nigeria and Uganda. Our findings provide insight on government policies and household and individual perceptions related to suppression of the virus, and consequences related to income loss, food security, education and the ability to access and purchase basic necessities. Although false beliefs about COVID-19 are prevalent, government action to limit the spread of the disease is associated with better knowledge about the disease and increased uptake of precautionary measures. We estimate that 256 million individuals, around $77 \%$ of the population across the four countries, live in households that have lost income since the start of the pandemic. Attempts to cope with this lost income are exacerbated by an inability to access medicine and staple foods for an estimated $30 \%$ of households who need these items. Income loss and lack of access to necessities have been borne disproportionately by households that were already impoverished before the pandemic. Finally, the loss of access to education has been profound, as the rate of maintaining student-teacher contact fell to an estimated $17 \%$ among households with children who were previously attending school.

When interpreting our results, several important considerations should be borne in mind. First, the global pandemic continues to develop and evolve at an extremely rapid pace. Consequently, data on the socioeconomic impacts of COVID-19, which take time to collect, clean, analyse and publish, are always at risk of becoming dated even before they are released. The World Bank LSMS programme, in partnership with the national statistical offices (NSOs) implementing the phone surveys, have worked to make the data available quickly. Even with this effort, publicly available data typically lags two months behind. Because of this lag, the picture that we present of the on-the-ground situation is likely to have changed in unexpected ways since the last round of data that we rely on was collected (August 2020). To this end, our results provide a perspective of the recent past that can be used by governments, policymakers and other stakeholders to direct resources during the pandemic as well as evidence for future research once the immediacy of the pandemic has passed. A second consideration is that, since the pandemic and data collect are ongoing, we do not yet have the complete picture of the socioeconomic impacts of COVID-19. Data collection will continue in each of these four countries for a full 12 months. Completion of the data collection will allow for a fuller picture of the effects of the pandemic on households in these low-income countries and the individuals therein. Before this happens, any evidence on the evolving effects of the virus on households, adults or children will be a snapshot of the situation at a particular time point.

Our study is also subject to several limitations. First and foremost, the results that we present are primarily descriptive in nature, relying on survey sampling weights and econometric methods common to the field to correct for selection bias. However, these corrections do not themselves establish a causal relationship between the onset of the pandemic and our outcomes of interest, and there may be remaining selection and non-response biases that the survey sampling weights and econometric methods fail to fully account for. Many adults in these countries were food insecure before the pandemic, and the loss of income or revenue may be due to events unrelated to the pandemic. More work is needed to align the post-COVID phone survey data with the pre-COVID face-to-face data in order to establish a pre- and post-pandemic panel. Second, our data comes from only four countries. The World Bank is in the process of conducting surveys in a number of other low-income countries, but these data were made publicly available only after this Article was written. We believe results from the countries considered here, which cover east, west, and southern Africa, are indicative of outcomes in neighbouring countries. However, the results are not in representative any statistical sense beyond the four countries the data come from.

Presently, it is not possible to reliably predict the trajectory of SARS-CoV-2 and its impacts within any given country. The precise morbidity and mortality burden and the economic benefits and drawbacks of different approaches to controlling the virus remain unknown. However, for the first time in 20 years, global extreme poverty is expected to rise, as the pandemic exacerbates the effects of climate change and conflict. An estimated 88 to 115 million people will be pushed into extreme poverty this year, with the total rising up to 150 million by $2021^{38}$. We have presented results on the economic burden of the global pandemic in low-income countries, where data have proved to be particularly scarce. Our findings point to a substantial scope for strengthening social protection systems to help cope with widespread income losses, persisting food insecurity and insufficient access to basic necessities. The results also highlight the need to sustain citizen-engagement efforts in order to promote health and behavioural measures against the spread of the disease and to dispel myths around COVID-19 that may have a bearing on the adoption of these safe practices ${ }^{39}$. Finally, there is room to build on rapidly evolving examples of large-scale, national efforts to increase access to technology in support of remote education $^{40}$. Going forward, understanding the contemporaneous socioeconomic impacts of the pandemic and the related restrictions on households, adults and children continue to be important for implementing policies and programmes to further curb adverse economic and health impacts.

\section{Methods}

Data. In each country, the NSO provides and implements the guidelines for the face-to-face and phone survey procedures, in collaboration with the World Bank LSMS and the Poverty and Equity Global Practice programmes. The only exception is in the case of Ethiopia, where the private firm implements the phone survey guidelines that are provided by the World Bank and are approved by the Central Statistical Agency of Ethiopia. The survey data are anonymized prior to public dissemination on the World Bank Microdata Library and our analysis relies on these anonymized public use datasets.

Phone survey data collection. The study uses high-frequency phone surveys on COVID-19 that were implemented in Ethiopia, Malawi, Nigeria and Uganda during the period May to August 2020, with support from the World Bank LSMS and the Poverty and Equity Global Practice. The selection of these countries was 
guided solely by the public availability of unit-record survey data at the time of the initiation of our research. Since then, additional phone survey rounds have been made publicly available for these four countries as well as Burkina Faso, Chad, Djibouti, Georgia, India, Kenya and Mali.

In each country, the phone survey aims to conduct monthly phone interviews, for a period of 12 months. The sample for these surveys is a national sample of households that had been interviewed during the latest round of the national longitudinal household survey implemented by the respective NSO, with financial and technical assistance from the World Bank LSMS-ISA initiative. See Supplementary Figs. 1 and 2 for more details on round-specific phone surveys response rates.

The implementing agency for the phone surveys in Ethiopia, Malawi, Nigeria and Uganda are Laterite Ethiopia, Malawi National Statistical Office, Nigeria Bureau of Statistics and Uganda Bureau of Statistics. The anonymized, unit-record phone survey data associated with each monthly survey round, together with the questionnaire, basic information document and interviewer manual for that round, are made publicly available within approximately four weeks of completion of phone interviews, through the World Bank Microdata Library, under the High-Frequency Phone Survey collection ${ }^{9-12}$ : https:// microdata.worldbank.org/index.php/catalog/hfps. The approach to the phone survey questionnaire design and sampling is comparable across countries. It is informed by the template questionnaire, the phone survey sampling guidelines and the computer-assisted telephone interviewing (CATI) guidelines that have been made publicly available by the World Bank. These can be accessed through the following links: template questionnaire, https://documents.worldbank.org/ en/publication/documents-reports/documentdetail/567571588697439581/ questionnaire-template; manual, https://documents.worldbank.org/en/ publication/documents-reports/documentdetail/431901588694657348/ interview-manual-for-template-questionnaire; sampling guidelines, https://documents.worldbank.org/en/publication/documents-reports/ documentdetail/742581588695955271/guidelines-on-sampling-design; and CATI guidelines, https://documents.worldbank.org/en/publication/documents-reports/ documentdetail/189691588696451053/guidelines-on-cati-implementation.

All regressions and results presented in this Article are based on the publicly available data from the World Bank Microdata Library. Data were downloaded from the website and processed using Stata 16.1. All cleaning, processing and analysis was conducted by the research team and the code has been made publicly available at Zenodo under accession 4568484.

\section{Sampling design for the pre-COVID-19 LSMS-ISA surveys. Ethiopia}

Socio-economic Survey 2018/19, IHPS 2019, GHS Panel 2018/19 and Uganda National Panel Survey 2019/20 serve as the sampling frame for the phone surveys that inform our analysis. We provide summary information on the sampling design for each survey in the Supplementary Information.

Food Insecurity Experience Scale. The estimates of prevalence of (1) moderate or severe and (2) severe food insecurity among adult individuals are based on the eight-question FIES, which was included as a module in the high-frequency phone survey. The FIES is an experience-based metric of food insecurity severity, which relies on people's direct responses to questions about their experiences with access to adequate food. This metric makes it possible to compare prevalence rates of food security across national and sub-national populations ${ }^{41}$. Widely used around the world, FIES is an appropriate measure for assessing food security as it is a direct measure of food insecurity which produces comparable estimates of food insecurity experienced by people in different contexts.

In the high-frequency phone surveys that inform our analyses, the FIES questions had a reference period of the last 30 days. Following the FIES standard survey model, eight questions were asked, aiming to capture whether the respondent or other adult households members: (1) were worried they would not have enough to eat, (2) were unable to eat healthy and nutritious food, (3) ate only a few kinds of foods, (4) had to skip a meal, (5) ate less than they thought they should, (6) ran out of food, (7) were hungry but did not eat, or (8) went without eating for a whole day.

The approach to process and analyse FIES data comes from item response theory ${ }^{42}$, also known as the Rasch model ${ }^{43}$, which accounts for the measurement of unobservable traits through the analysis of responses to survey. Analysis of FIES data involves parameter estimation, statistical validation and calculation of individual and population prevalence estimates, as appropriate, for food insecurity. In this analysis, a respondent's raw score (an integer between zero and eight) is determined. On the basis of this raw score, an interval measure of the severity of food security based on global standards is determined by equating (calibrating the score on a common metric) ${ }^{44}$.

Equating is completed for each household, which allows us to estimate the prevalence of (1) moderate or severe and (2) severe food insecurity among adult household members. Following procedures detailed by the Food and Agriculture Organization of the United Nations and the associated programme, Voices of the Hungry, we calculate FIES for households in our data as follows:

1. Beginning with data files constructed with Stata, we construct binary variables for each of the eight FIES components that take a value of 1 for 'yes' and 0 for 'no' answers (and leave untouched any missing answers). We reorder the variables and recode the variable names to ensure the following order and naming convention: Worried, Healthy, FewFood, Skipped, AteLess, RunOut, Hungry and WhlDay.

2. We construct adult population weights $\left(w_{\mathrm{a}}\right)$, by multiplying household sampling weights $\left(w_{(i, \text { final })}\right)$ with the count of adult members in each household. This household sampling weight is retained. The resulting file is exported to .csv format. We upload the .csv file to the FIES Shiny app: https://fies.shinyapps.io/ExtendedApp/, which was developed by the Food and Agriculture Organization of the United Nations, the custodian agency for FIES ${ }^{45}$.

3. In the app, under the 'Item and Raw Score Stat' tab, we check the Infit statistic for each component. If a component is not within the bounds 0.7 and 1.3 , it is dropped from the analysis. At least six questions should be retained in this step.

4. We equate the included items to the global standard. It is sometimes the case that some items may diverge from the global standard to be included in the calibration of the country scale to the global standard. The rule then is to exclude items that differ by more than 0.35 . This is accomplished in the 'Equating' tab. If there are multiple items differing by more than 0.35 in our sample, we iterate on which set of items to exclude. At least five items must be retained for a robust estimation. The items that are excluded from the equating procedure are not dropped entirely from the construction of the FIES indicators but are ignored when equating the country scores to the global standard.

5. Under the 'Additional Information' tab, we next 'download respondent-level model-based variables' This provides a dataset with as many rows as the .csv file that was uploaded into the Shiny app in step 2. This downloaded dataset includes the estimates of prevalence of (1) moderate or severe and (2) severe food insecurity among adult individuals in accordance with the raw score for each household (that is, the count of 'yes' answers across the eight questions).

6. We import the data downloaded in step 5 into Stata and using raw score as the linking variable and merge it with other Stata-formatted data files that are defined at the household-level.

Consumption quintiles. To define pre-COVID-19 household per capita consumption quintiles, we rely on the pre-public-dissemination versions of the consumption aggregates. These are provided by the LSMS team on an exceptional basis, with clearance from the respective NSOs, for the pre-COVID-19 LSMSISA-supported surveys that serve as a sampling frame for the high-frequency phone surveys on COVID-19. In the case of Ethiopia, Nigeria and Malawi, the consumption aggregates are computed by the respective NSO, with technical assistance from the LSMS. In the case of Uganda, the consumption aggregate is computed by and has been obtained from the Uganda Bureau of Statistics. In the specific case of Malawi, the consumption aggregate is for IHPS 2016, as this information is unavailable for IHPS 2019.

Econometrics. Calculating phone survey sampling weights. As we rely on phone interviews with a sample of households that had been interviewed face-to-face prior to the COVID-19 pandemic as part of the LSMS-ISA-supported national longitudinal household survey, there are two potential sources of bias in the resulting data: (1) selection bias associated with not being able to call LSMS-ISA households that do not own mobile phones and (2) non-response bias associated with not being able to interview households that are targeted for phone interviews. Without correcting for these two sources of bias, results of our analysis are likely to underestimate the negative effects of COVID-19, as poorer households are less likely than wealthier households to have phones, and less likely to respond even if they do have phones.

In our analysis, we use the phone survey weights that are provided in public use datasets and that are computed in an attempt to address the potential sources of bias using well-established methods ${ }^{13,15}$. The phone survey weights in each country build on the sampling weights for the associated LSMS-ISA-supported survey. They are calibrated to address the selection bias introduced from LSMS-ISA households not owning a mobile phone and non-response bias from not answering the phone. The non-response bias is overwhelmingly due to non-working phone numbers or prospective respondents not answering calls (as opposed to refusals). To calculate survey weights for the phone survey, we implement the following steps in Ethiopia, Malawi and Uganda ${ }^{14}$.

1. We begin with the existing sampling weight for each household, as computed for the associated LSMS-ISA-supported survey $\left(w_{i, \text { pre }}\right)$, which are available in those datasets.

2. We calculate the probability of selection into the phone survey for each household $\left(p_{i}\right)$, as the total number of LSMS-ISA households for which contact was attempted in that household's region $\left(m_{r}\right)$ divided by the total number of LSMS-ISA households in that region $\left(M_{r}\right)$ :

$$
p_{i}=\frac{m_{r}}{M_{r}} .
$$

3. We calculate the adjusted household sampling weight $\left(w_{i, \text { phone }}\right)$ by multiplying the existing LSMS-ISA sampling weight with the reciprocal of the probability of selection in the phone survey, as calculated in step 2: 


$$
w_{i, \text { phone }}=w_{i, \text { pre }} \frac{1}{p_{i}} .
$$

4. Using the entire LSMS-ISA sample, we run a multivariate logistic regression in which the dependent variable (phone) is a binary variable that is equal to 1 for LSMS-ISA households that were successfully interviewed for the phone survey and equal to 0 otherwise. The independent variables $\left(X_{k}\right)$ included represent a range of household, dwelling and head of household attributes that predict the likelihood of a completed phone survey interview:

$$
P(\text { phone }=1)=F\left(\beta_{0}+\sum_{k=1}^{K} \beta_{k} X_{k}\right) .
$$

5. The independent variables included vary by country and round, since the sampling frame and survey instrument vary by country and round. The basic information document for each phone survey provides specifics of what variables are included.

6. We predict the probability of response (that is propensity score) and create ten equal groups (deciles) for this variable. Within each decile $d$, we compute the mean value and take the reciprocal as the phone survey attrition correction factor for observations in each decile $\left(\mathrm{ac}_{D=d}\right)$ :

$$
\mathrm{ac}_{D=d}=\frac{1}{\left(\frac{\sum_{i=1}^{N} \widehat{\mathrm{phone}_{\mathrm{i}}}}{N}\right)}
$$

7. where $\widehat{\text { phone }}_{i}$ is the predicted value (that is propensity score) for each observation $i$ and $N$ is the total number of individuals in each decile. We then apply the attrition correction factor to the adjusted household sampling weight as derived in step 3:

$$
w_{i, \text { ac }}=\operatorname{ac}_{D=d} \times w_{i, \text { phone }} .
$$

8. We winsorize the resulting weight $\left(w_{i, a c}\right)$ by replacing the top $2 \%$ of observations, with the value at the 98 th percentile used as the cut-off point.

9. Finally, we post-stratify weights to reduce standard errors and to match the projected population totals at the highest spatial resolution possible, ranging from region to district, on the basis of the data availability in each country. Population projections come from NSOs, often as part of census data. The post-stratification weights $\left(w_{\mathrm{ps}}\right)$ are calculated as the weighted total number of observations from the data divided by the census projections:

$$
w_{\mathrm{ps}}=\frac{\sum_{i=1}^{M_{r}} w_{i, \mathrm{ac}}}{\text { population }}
$$

where $M_{r}$ is the total population in the region, or whatever is the highest spatial resolution available, and population is the NSO's projected population for that region. The final weight is then calculated as:

$$
w_{i, \text { final }}=w_{\mathrm{ps}} \times w_{i, \mathrm{ac}} .
$$

In our case, post-stratification is not a major adjustment to the weights but rather a fine tuning of their values that enables us to ensure that the population estimates that are obtained through the summation of the sampling weights match with the official population projections at the time of the phone survey. This is a standard calibration that is also applied in the calculation of both longitudinal and cross-sectional face-to-face surveys, including the pre-COVID-19 LSMS-ISA surveys that have been used as sampling frames for our phone surveys.

In the case of Nigeria, to obtain a nationally representative sample for the phone survey, a subsample of around 1,800 interviewed households was targeted. On the basis of the experience with prior phone surveys in Nigeria, a response rate of $60 \%$ was assumed, implying a required number of 3,000 households be contacted in order to reach the target subsample of 1,800 . These households were selected from the frame of 4,934 GHS panel households with at least one phone number for a household member or a reference individual. Given the auxiliary information available in the GHS panel for households, a balanced sampling approach was adopted using the cube method ${ }^{46}$. This approach enables selection of a random sample that still retains the properties of the pre-COVID sampling frame across selected covariates. Balancing on these variables reduces the variance of the resulting estimates, assuming that the selected covariates are correlated with the variable of interest. Calibration to the balancing variables after the data collection further reduces this variance ${ }^{15}$. The sample was balanced across state, sector (urban or rural), household size, per capita consumption expenditure, household head sex and education, and household ownership of a mobile phone.

Econometric estimation. The econometric methods applied here describe behaviour of the various outcomes of interest in the data (for example food security, education and income loss). This setup allows us to make inferences and conduct more complex statistical tests with multiple controls for differences across populations than simple univariate tests for differences in means ${ }^{16}$. For a given outcome, we regress that outcome on a set of covariates, which are held fixed. In our case, the estimated value, calculated using ordinary least squares (OLS), is the mean in the population-weighted sample surveyed by the high-frequency phone surveys on COVID-19. Given that the calculated weights correct for selection bias and non-response bias, this common OLS approach allows us to make unbiased inference about the impact of a specific covariate on the outcome for the national population ${ }^{16}$. To ensure correct inference of statistical tests, we calculate HuberWhite robust standard errors, which correct for heteroskedasticity. Our approach to the weighted OLS estimation allows us to produce unbiased estimates of impacts of covariates on the population as a whole without explicitly describing the underlying mechanisms.

Reporting Summary. Further information on research design is available in the Nature Research Reporting Summary linked to this article.

\section{Data availability}

The data used in this study can be freely downloaded from the World Bank Microdata Library. All data used in this study can be downloaded from the cited sources. Specific country datasets are contained in refs. ${ }^{9-12}$.

\section{Code availability}

The code used to generate these analyses is available at Zenodo under accessions 4568484 and 4060416. The code is licensed under the MIT license.

Received: 18 October 2020; Accepted: 5 March 2021;

Published online: 30 March 2021

\section{References}

1. WHO. Coronavirus Disease (COVID-19) Weekly Epidemiological Update (24 November 2020); https://www.who.int/publications/m/item/ weekly-epidemiological-update-24-november-2020

2. Hsiang, S. et al. The effects of large-scale anti-contagion policies on the COVID-19 pandemic. Nature 584, 262-267 (2020).

3. Haug, N. et al. Ranking the effectiveness of worldwide COVID-19 government interventions. Nat. Hum. Behav. 4, 1303-1312 (2020).

4. Global Economic Prospects June 2020 (World Bank, 2020); https://doi. org/10.1596/978-1-4648-1553-9

5. Walker, P. G. T. et al. The impact of COVID-19 and strategies for mitigation and suppression in low- and middle-income countries. Science 369, 413-422 (2020).

6. Mbow, M. et al. COVID-19 in Africa: dampening the storm? Science 369, 624-626 (2020).

7. Laborde, D. et al. COVID-19 risks to global food security. Science 369, 500-502 (2020)

8. Morsey, H., Balma, L. \& Mukasa A. N. 'Not a Good Time': Economic Impact of COVID-19 in Africa Working Paper Series no. 338 (African Development Bank Group, 2020).

9. High-Frequency Phone Survey on COVID-19 2020-Ethiopia (World Bank, 2020); https://microdata.worldbank.org/index.php/catalog/3716

10. High-Frequency Phone Survey on COVID-19-Malawi (World Bank, 2020); https://microdata.worldbank.org/index.php/catalog/3766

11. High-Frequency Phone Survey on COVID-19 2020-Nigeria (World Bank, 2020); https://microdata.worldbank.org/index.php/catalog/3712

12. High-Frequency Phone Survey on COVID-19 2020-Uganda (World Bank, 2020); https://microdata.worldbank.org/index.php/catalog/3765

13. Cochran, W. G. Sampling Techniques 3rd edn (Wiley, 1977).

14. Himelein, K. Weight calculations for panel surveys with subsampling and split-off tracking. Stat. Public Policy 1, 40-45 (2014).

15. Tillé, Y. Sampling Algorithms (Springer, 2006).

16. Greene, W. H. Econometric Analysis 8th edn (Pearson, 2017).

17. Angrist, J. D. \& Pischke, J.-S. Mostly Harmless Econometrics (Princeton Univ. Press, 2009).

18. Khamsi, R. If a coronavirus vaccine arrives, can the world make enough? Nature 580, 578-580 (2020).

19. WHO Calls for Equitable Access to Future COVID-19 Vaccines in Africa (WHO, 2020); https://www.afro.who.int/news/ who-calls-equitable-access-future-covid-19-vaccines-africa

20. PERC: Brief on Public Health and Social Measure Implementation in Africa (Africa CDC, 2020); https://africacdc.org/download/ perc-brief-on-public-health-and-social-measure-implementation-in-africa/ 21. Khodr, A. With schools closed due to COVID-19, Ethiopia grapples with providing distance education to children. UNICEF https://www.unicef.org/ ethiopia/stories/schools-closed-due-covid-19-ethiopia-grapples-providi ng-distance-education-children (2020).

22. A State of Emergency Proclamation Enacted to Counter and Control the Spread of COVID-19 and Mitigate Its Impact Proclamation 3/2020 (Government of Ethiopia, 8 April 2020); https://www.ilo.org/dyn/natlex/natlex4. detail?p_isn=110046\&p_lang=en 
23. Address by H. E. Muhammadu Buhari, President of the Federal Republic of Nigeria on the COVID-19 pandemic https://www.nigerianembassy.ru/index. php/address-buhari-about-covid19.html (30 March 2020).

24. Buhari extends Lagos, Ogun, FCT lockdown by two weeks. The Nation https://thenationonlineng.net/full-text-of-president-buharis-address/ (14 April 2020).

25. Museveni, Y. Address on the corona virus (COVID19.) guidelines on avoiding the pandemic. Official website for Yoweri K. Museveni, President of Uganda https://www.yowerikmuseveni.com/address-corona-virus-covid19-guidelines-avoiding-pandemic (2020).

26. Museveni, Y. More guidelines on COVID19, preventive measures and the need for a shut down. Official website for Yoweri K. Museveni, President of Uganda https://www.yowerikmuseveni.com/more-guidelines-covid 19-preventive-measures-and-need-shut-down (2020).

27. Mutharika, A. P. Declaration of State of Disaster by Malawi President Peter Mutharika. United Nations Malawi https://malawi.un.org/en/ 46778-declaration-state-disaster-malawi-president-peter-mutharika (20 March 2020).

28. Mutharika, A. P. Speech by President Mutharika on 21-day Covid-19 lockdown. United Nations Malawi https://malawi.un.org/en/467 97-speech-president-mutharika-21-day-covid-19-lockdown (14 April 2020).

29. Order Granting Permission to Apply for Judicial Review and Interlocutory Order of Injunction Judicial Review Cause No. 22 of 2020 https://perma.cc/ MML4-8FNU (High Court of Malawi, 2020).

30. Gallotti, R. et al. Assessing the risks of 'infodemics' in response to COVID-19 epidemics. Nat. Hum. Behav. 4, 1285-1293 (2020).

31. ILO Monitor: COVID-19 and the World of Work 6th edn (ILO, 2020).

32. Gunderson, C. et al. Food insecurity during COVID-19. Appl. Econ. Perspect. Policy 43, 153-161 (2021).

33. Toffolutti, V. et al. Is the COVID-19 pandemic turning into a European food crisis? Eur. J. Public Health 30, 626-627 (2020).

34. Ahn, S. \& Norwood, F. B. Measuring food insecurity during the COVID-19 pandemic of spring 2020. Appl. Econ. Perspect. Policy 43, 162-168 (2021).

35. Ahmed, F. et al. Why inequality could spread COVID-19. Lancet Public Health 5, e240 (2020).

36. Laborde, D., Martin, W. \& Vos, R. Poverty and food insecurity could grow dramatically as COVID-19 spreads. IFPRI Blog https://www.ifpri.org/blog/ poverty-and-food-insecurity-could-grow-dramatically-covid-19-spreads (2020).

37. The Millennium Development Goals Report 2015 (United Nations, 2015).

38. World Bank Group's Operational Response to COVID-19 (Coronavirus) Projects List (World Bank, 2020); https://www.worldbank.org/en/about/ what-we-do/brief/ world-bank-group-operational-response-covid-19-coronavirus-projects-list

39. Update on IDA Contribution to COVID-19 Pandemic Response (English) (World Bank, 2020); https://documents.worldbank.org/en/publication/ documents-reports/documentdetail/673321588557820754/ update-on-ida-contribution-to-covid-19-pandemic-response

40. Remote Learning, EdTech and COVID-19 (World Bank, 2020); https://www. worldbank.org/en/topic/edutech/brief/edtech-covid-19
41. Voices of the Hungry: Bringing Experience-based Food Insecurity Measurement to the Global Level (FAO, 2019); http://www.fao.org/in-action/ voices-of-the-hungry/background/en/

42. Nord, M. Introduction to Item Response Theory applied to Food Security Measurement: Basic Concepts, Parameters, and Statistics http://www.fao. org/3/a-i3946e.pdf (FAO, 2014).

43. Wright, B. D. Solving Measurement Problems with the Rasch Model (Univ. of Chicago, 1977).

44. The Food Insecurity Experience Scale: Measuring Food Insecurity Through People's Experiences (FAO, 2017); http://www.fao.org/3/a-i7835e.pdf

45. Using the FIES App: A Simple Tool for the Analysis of Food Insecurity Experience Scale Data (FAO, 2020); http://www.fao.org/3/ca9318en/ca9318en.pdf

46. Deville, J.-C. \& Tillé, Y. Efficient balanced sampling: the cube method. Biometrika 91, 893-912 (2004).

\section{Acknowledgements}

Funding for data collection and analysis comes from the World Bank Multi-Donor Trust Fund for Integrated Household and Agricultural Surveys in Low and Middle-Income Countries (TF072496). The funders had no role in study design, data collection and analysis, decision to publish, or preparation of the manuscript. We acknowledge the research assistance provided by A. Furbush and J. Brubaker. We thank the individuals involved in the design, implementation and dissemination of high-frequency phone surveys on COVID-19, specifically the World Bank LSMS team, and the phone survey managers and interviewers at the Malawi National Statistical Office, the Nigeria Bureau of Statistics, the Uganda Bureau of Statistics and Laterite Ethiopia.

\section{Author contributions}

Authors are listed alphabetically. A.J.: conceptualization, formal analysis, data curation, writing of original draft and visualization. T.K.: conceptualization, investigation, writing, review, editing and visualization. J.D.M.: conceptualization, formal analysis, data curation, writing of original draft and visualization. All authors contributed to the final draft.

\section{Competing interests}

The authors declare no competing interests.

\section{Additional information}

Extended data is available for this paper at https://doi.org/10.1038/s41562-021-01096-7. Supplementary information The online version contains supplementary material available at https://doi.org/10.1038/s41562-021-01096-7.

Correspondence and requests for materials should be addressed to J.D.M.

Peer review information Nature Human Behaviour thanks the anonymous reviewers for their contribution to the peer review of this work.

Reprints and permissions information is available at www.nature.com/reprints.

Publisher's note Springer Nature remains neutral with regard to jurisdictional claims in published maps and institutional affiliations.

(c) The Author(s), under exclusive licence to Springer Nature Limited 2021 


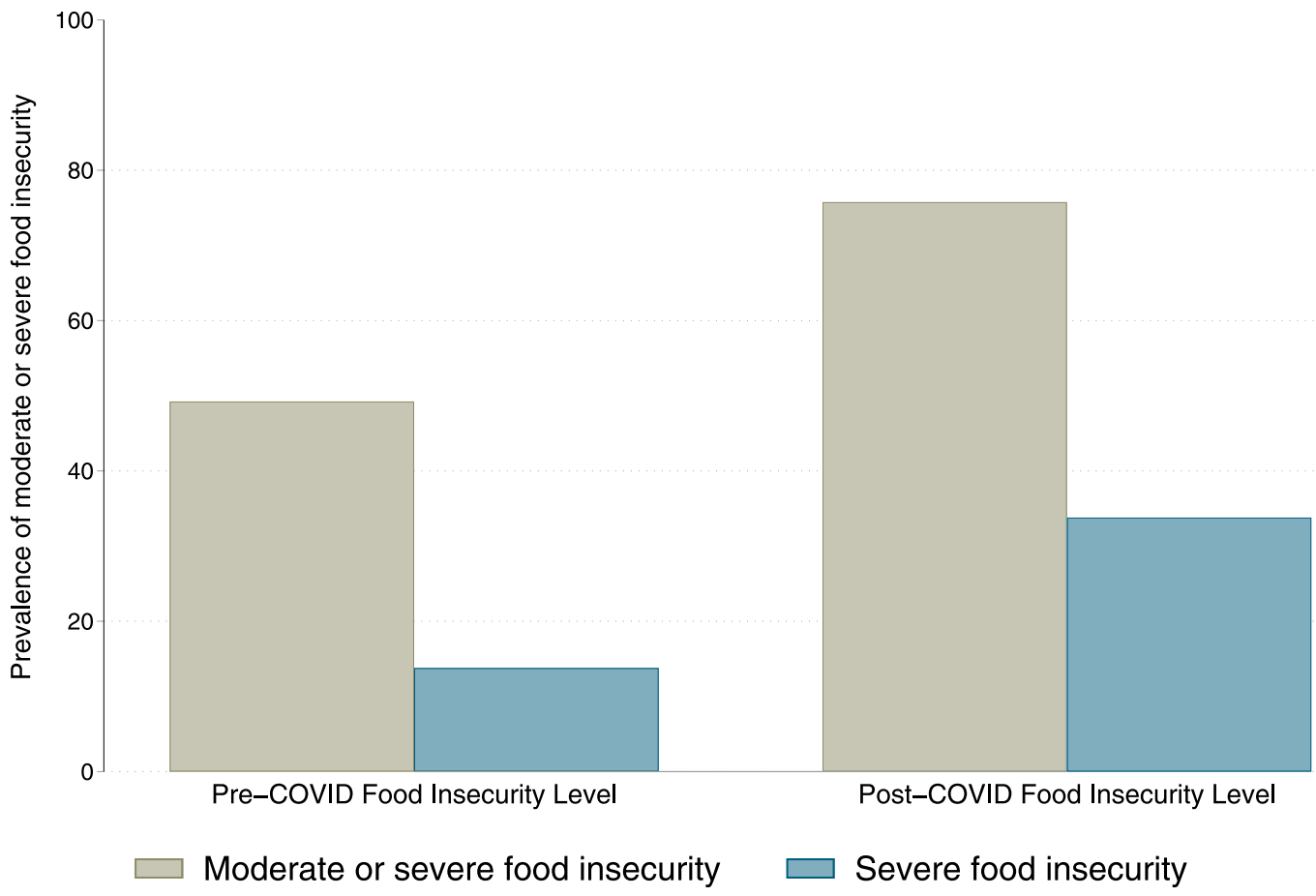

Extended Data Fig. 1 | Changes in food security in Nigeria, pre- and post-pandemic onset. Prevalence of moderate and/or severe food insecurity among adult individuals in Nigeria, pre- and post-pandemic. Pre-pandemic data comes from a post-planting visit between July and September of 2019. Post-pandemic data comes from the Nigeria COVID phone surveys conducted between May and August of $2020(n=1,819)$. 


\section{Reporting Summary}

Nature Research wishes to improve the reproducibility of the work that we publish. This form provides structure for consistency and transparency in reporting. For further information on Nature Research policies, see our Editorial Policies and the Editorial Policy Checklist.

\section{Statistics}

For all statistical analyses, confirm that the following items are present in the figure legend, table legend, main text, or Methods section.

n/a Confirmed

$\bigotimes$ The exact sample size $(n)$ for each experimental group/condition, given as a discrete number and unit of measurement

$\square$ A statement on whether measurements were taken from distinct samples or whether the same sample was measured repeatedly

$\square$ The statistical test(s) used AND whether they are one- or two-sided

$\square$ Only common tests should be described solely by name; describe more complex techniques in the Methods section.

$\square$ A description of all covariates tested

$\square$ \A description of any assumptions or corrections, such as tests of normality and adjustment for multiple comparisons

$\square$ A full description of the statistical parameters including central tendency (e.g. means) or other basic estimates (e.g. regression coefficient)

$\triangle$ AND variation (e.g. standard deviation) or associated estimates of uncertainty (e.g. confidence intervals)

$\varnothing$ For null hypothesis testing, the test statistic (e.g. $F, t, r$ ) with confidence intervals, effect sizes, degrees of freedom and $P$ value noted

Give P values as exact values whenever suitable.

Х $\square$ For Bayesian analysis, information on the choice of priors and Markov chain Monte Carlo settings

$\square$ \ For hierarchical and complex designs, identification of the appropriate level for tests and full reporting of outcomes

$\square \bigotimes$ Estimates of effect sizes (e.g. Cohen's $d$, Pearson's $r$ ), indicating how they were calculated

Our web collection on statistics for biologists contains articles on many of the points above.

\section{Software and code}

Policy information about availability of computer code

Data collection Computer code was not used to collect data.

Data analysis Stata MP 16.1

For manuscripts utilizing custom algorithms or software that are central to the research but not yet described in published literature, software must be made available to editors and reviewers. We strongly encourage code deposition in a community repository (e.g. GitHub). See the Nature Research guidelines for submitting code \& software for further information.

\section{Data}

Policy information about availability of data

All manuscripts must include a data availability statement. This statement should provide the following information, where applicable:

- Accession codes, unique identifiers, or web links for publicly available datasets

- A list of figures that have associated raw data

- A description of any restrictions on data availability

The data used in this study can be freely downloaded from the World Bank Microdata Library. DOls and links to each data set are provided in the manuscript. 


\section{Field-specific reporting}

Please select the one below that is the best fit for your research. If you are not sure, read the appropriate sections before making your selection.

Life sciences $\quad \square$ Behavioural \& social sciences $\square$ Ecological, evolutionary \& environmental sciences

For a reference copy of the document with all sections, see nature.com/documents/nr-reporting-summary-flat.pdf

\section{Behavioural \& social sciences study design}

All studies must disclose on these points even when the disclosure is negative.

Study description

The study analyzes longitudinal survey data from four African countries with varying policy implementations to estimate the impact of the impact of COVID-19 on socioeconomic indicators for households, individuals, and children. The data sources include preCOVID-19 national face-to-face surveys and monthly phone surveys that were implemented following the onset of the pandemic.

Research sample The longitudinal survey data informing our analysis stem from (i) the pre-COVID-19 national face-to-face household surveys that were designed to be representative in each country at the national, urban/rural and regional-levels, and (ii) the monthly phone surveys that aimed to re-interview all households that had previously been interviewed by the aforementioned pre-COVID-19 faceto-face household surveys and that had provided at least one mobile phone number for a household member or a reference individual outside the household. Please see the appendix for more information on how the phone survey samples were drawn.

Sampling strategy

Please see the appendix that details the sampling strategy for the monthly phone surveys as well as the approach to calculation of the phone survey sampling weights that help provide general household population-representative insights.

Data collection Data collection is detailed in the appendix and the anonymized unit-record survey data are publicly available on the World Bank Microdata Library.

Timing

Data collection began in May 2021 and completed in July 2021.

Data exclusions

Data was excluded only if the record was incomplete or missing information on the relevant variables.

Non-participation

Participants either declined to be interviewed as part of the informed consent request or they were not reachable by phone. Details of unreached participants and correction for potential attrition and selection bias are discussed in the appendix.

Randomization

Randomization was not possible since the study examines the impact of COVID-19 and related national policies.

\section{Reporting for specific materials, systems and methods}

We require information from authors about some types of materials, experimental systems and methods used in many studies. Here, indicate whether each material, system or method listed is relevant to your study. If you are not sure if a list item applies to your research, read the appropriate section before selecting a response.

Materials \& experimental systems

$\mathrm{n} / \mathrm{a}$ Involved in the study

\ $\square$ Antibodies

$\bigotimes \square$ Eukaryotic cell lines

\ $\square$ Palaeontology and archaeology

$\bigotimes \square$ Animals and other organisms

$\square \bigotimes$ Human research participants

$\bigotimes \square$ Clinical data

$\bigotimes \square$ Dual use research of concern

\author{
Methods \\ $\mathrm{n} / \mathrm{a}$ Involved in the study \\ \ $\square$ ChIP-seq \\ Х $\square$ Flow cytometry \\ \ $\square$ MRI-based neuroimaging
}

\section{Human research participants}

\section{Policy information about studies involving human research participants}

Population characteristics

Recruitment
Please see the above response on the research sample. For more details, please consult the appendix.

In each country, the monthly phone survey aimed to re-interview all households that had previously been interviewed by a pre-COVID-19 national face-to-face household survey and that had provided at least one mobile phone number for a household member or a reference individual outside the household. The face-to-face and phone surveys are conducted by the respective national statistical office (NSO) in each country, in accordance with the Statistical Act. The only exception is in the case of the phone survey in Ethiopia, which is conducted by a private firm contracted by the World Bank (namely Laterite Ethiopia) but upon clearance from the Central Statistical Agency (CSA) of Ethiopia, as the survey implementing agency for the 
face-to-face survey that served as the sampling frame for the phone survey. The face-to-face and phone survey data collection is conducted in each country with the informed consent of each survey respondent.

Ethics oversight

In each country, the NSO provides and implements the guidelines for the face-to-face and phone survey procedures, in collaboration with the World Bank Living Standards Measurement Study (LSMS) Program and the Poverty and Equity Global Practice. The only exception is again in the case of Ethiopia, where the private firm implements the phone survey guidelines that are provided by the World Bank and are approved by the CSA of Ethiopia. The survey data are anonymized prior to public dissemination on the World Bank Microdata Library and our analysis relies on these anonymized public use datasets.

Note that full information on the approval of the study protocol must also be provided in the manuscript. 